\title{
Keeping pace with the world: improving the clinical practice of anti-NMDAR encephalitis in China
}

\section{Hongzhi Guan}

Department of Neurology, Peking Union Medical College Hospital, Chinese Academy of Medical Sciences and Peking Union Medical College, Beijing, China

Correspondence to: Hongzhi Guan, MD. Department of Neurology, Peking Union Medical College Hospital, Chinese Academy of Medical Sciences and Peking Union Medical College, No. 1 Shuaifuyuan, Wangfujing, Dongcheng District, Beijing 100730, China. Email: guanhz@263.net.

Provenance and Peer Review: This article was commissioned and reviewed by the Section Editor Dr. Jinming Han (Department of Clinical Neuroscience, Center for Molecular Medicine, Karolinska Institutet, Karolinska University Hospital, Stockholm, Sweden).

Response to: Ceanga M, Chung HY, Geis C. Anti-NMDA receptor encephalitis: epidemiological differences and common challenges. Ann Transl Med 2020;8:716.

Desestret V. The Chinese experience with anti-NMDAR encephalitis. Ann Transl Med 2020;8:718.

Submitted Apr 01, 2020. Accepted for publication Jul 13, 2020.

doi: 10.21037/atm-2020-76

View this article at: http://dx.doi.org/10.21037/atm-2020-76

The phenomenon and significance of low frequency of paraneoplastic cases in our Chinese cohort of anti-NMDAR encephalitis deserves clinical attention. Only $19.5 \%$ of the patients in our cohort of 220 cases had a tumor. But the fact that $29.4 \%$ of female patients had an ovarian teratoma warrants more oncological evaluation in the subgroup of young female (1). Other Chinese studies have reported even lower prevalence of tumors $(6.7-8.1 \%)$ which may contribute to a relatively higher relapsing rate in these cohorts as the absence of tumor is a risk factor for relapses (2).

Whether the difference in oncological data indicates possibility of difference trigger factors and source of peripheral autoantigens remains a question. Except for a small portion of patients with post-HSE anti-NMDAR encephalitis, most patients, either tumor-positive or tumornegative, have typical presentation and course of antiNMDAR encephalitis. As for these tumor-negative cases in Asian and Chinese cohorts, to identify a potential source of autoantigens is theoretically reasonable and clinically practical. That is why we screen prominent melanocytic nevi in tumor-negative cases with refractory or relapse course $(3,4)$.

The frequency of paraneoplastic cases is expected to decline as more cohorts of anti-NMDAR encephalitis accumulated. In fact, the decline in proportion of paraneoplastic cases will always be experienced in the series study of paraneoplastic encephalitis. AntiNMDAR encephalitis was first described by Dalmau as a paraneoplastic autoimmune encephalitis (AE) in 2007 (5). Then Dalmau et al. described the clinical characteristics of 100 patients and reported that $59 \%$ of patients had tumors in 2008 (6). When Titulaer et al. reported the long-term outcome of more than 500 patients in 2013, the prevalence of an underlying neoplasm decreased to $38 \%$ (2). The research processes to discovery novel antineuronal antibodies emphasize on describing a novel neurological syndrome, usually the paraneoplastic one as the first promising step. Clinical indication of novel antibodies test tends to be more restricted to typical cases including paraneoplastic cases before a fully opening availability of the test.

According to our cohort and experience, re-enforced first-line immunotherapy based on repeated first-line therapy is effective with a good short-term outcome. Our team had respectively analyzed the immunotherapy strategy for 35 cases with severe anti-NMDAR mostly hospitalized to ICU in PUMCH from 2011 to 2015. All cases received intravenous immunoglobulin (IVIg), for one to a maximum of seven cycles, with an average of three cycles; $91.43 \%$ of cases received glucocorticoid therapy and $42.86 \%$ received long-term MMF therapy as add-on therapy for refractory course. Only $14.29 \%$ received second-line therapy 
including rituximab and/or intravenous cyclophosphamide. All cases improved and were transferred out from ICU, the median length of stay in ICU was 6 weeks and the median duration of hospitalization was around ten weeks. Although the add-on immunotherapy with MMF as an alternative to rituximab warranted further study, the re-enforced firstline immunotherapy based on multicycle IVIg also achieved good long-term prognoses in other Chinese cohorts of severe anti-NMDAR encephalitis (7).

To some extent, the treatment strategy of AE will still depend on different centers in different countries. However, we can expect a better prognosis in future studies from different centers. Antibodies panel related to $\mathrm{AE}$ is among the first-line tests in many neurological centers in China which contributes to a more prompt and efficient immunotherapy. Increasing cases have enriched neurologists' experience in treating AE. Encephalitis has been considered as a novel subspecialty and encephalitis clinics or encephalitis centers have been established by the neurologists across China.

\section{Acknowledgments}

Funding: None.

\section{Footnote}

Conflicts of Interest: The author has completed the ICMJE uniform disclosure form (available at http://dx.doi. org/10.21037/atm-2020-76). The author has no conflicts of interest to declare.

Ethical Statement: The author is accountable for all aspects of the work in ensuring that questions related to the accuracy or integrity of any part of the work are appropriately investigated and resolved.

Cite this article as: Guan H. Keeping pace with the world: improving the clinical practice of anti-NMDAR encephalitis in China. Ann Transl Med 2020;8(15):976. doi: 10.21037/atm2020-76
Open Access Statement: This is an Open Access article distributed in accordance with the Creative Commons Attribution-NonCommercial-NoDerivs 4.0 International License (CC BY-NC-ND 4.0), which permits the noncommercial replication and distribution of the article with the strict proviso that no changes or edits are made and the original work is properly cited (including links to both the formal publication through the relevant DOI and the license). See: https://creativecommons.org/licenses/by-nc-nd/4.0/.

\section{References}

1. Xu X, Lu Q, Huang Y, et al. Anti-NMDAR encephalitis: A single-center, longitudinal study in China. Neurol Neuroimmunol Neuroinflamm 2019;7:e633.

2. Titulaer MJ, McCracken L, Gabilondo I, et al. Treatment and prognostic factors for long-term outcome in patients with anti-NMDA receptor encephalitis: an observational cohort study. Lancet Neurol 2013;12:157-65.

3. Yin $\mathrm{H}, \mathrm{Zhu} \mathrm{C}$, Ren H, et al. Resection of melanocytic nevi as a potential treatment of anti-NMDAR encephalitis patients without tumor: report of three cases. Neurol Sci 2018;39:165-7.

4. Yang XZ, Cui LY, Ren HT, et al. Anti-NMDAR encephalitis after resection of melanocytic nevi: report of two cases. BMC Neurol 2015;15:165.

5. Dalmau J, Tüzün E, Wu HY, et al. Paraneoplastic anti-Nmethyl-D-aspartate receptor encephalitis associated with ovarian teratoma. Ann Neurol 2007;61:25-36.

6. Dalmau J, Gleichman AJ, Hughes EG, et al. Anti-NMDAreceptor encephalitis: case series and analysis of the effects of antibodies. Lancet Neurol 2008;7:1091-8.

7. Zhang $Y$, Liu G, Jiang $M$, et al. Clinical Characteristics and Prognosis of Severe Anti-N-methyl-D-aspartate Receptor Encephalitis Patients. Neurocrit Care 2018;29:264-72. 